\title{
Evolution of the symbiotic nova RX Puppis
}

\author{
J. Mikołajewska*, E. Brandi ${ }^{\dagger}$, L. Garcia ${ }^{\dagger}$, O. Ferrer ${ }^{\dagger}$, C. Quiroga ${ }^{\dagger}$ and G.C. \\ Anupama** \\ ${ }^{*}$ N. Copernicus Astronomical Center, Bartycka 18, 00716 Warsaw, Poland \\ mikolaj@camk.edu.pl \\ ${ }^{\dagger}$ Facultad de Ciencias Astronómicas y Geofisicas, UNLP - CIC - CONICET, 1900 La Plata, \\ Argentina \\ ** Indian Institute of Astrophysics, Bangalore 560034, India
}

\begin{abstract}
We present and discuss a hundred year history of activity of the hot component of RX Pup based on optical photometry and spectroscopy. The outburst evolution of RX Pup resembles that of other symbiotic novae whereas at quiescence the hot component shows activity (high and low activity states) resembling that of symbiotic recurrent novae T CrB and RS Oph.
\end{abstract}

\section{INTRODUCTION}

RX Pup is a long-period interacting binary system consisting of a Mira variable pulsating with $\mathrm{P}=578$ days, surrounded by a thick dust shell, and a hot white dwarf companion accreting material from the Mira's wind. The binary separation could be as large as $a \geq 50$ a.u. (corresponding to $P_{\mathrm{orb}} \geq 200 \mathrm{yr}$ ) as suggested by the permanent presence of a dust shell around the Mira component ([[])).

The analysis of multifrequency observations by [1] has shown that most, if not all, photometric and spectroscopic activity of RX Pup in the UV, optical and radio range is due to activity of the hot component, while the Mira variable and its circumstellar environment is responsible for practically all changes in the IR range (Fig.1). In particular, RX Pup underwent a nova-like eruption during the last three decades. The evolution of the hot component in the HR diagram (Fig.11 of [1]) as well as evolution of the nebular emission in 1970-1993 is consistent with a symbiotic nova eruption, with the luminosity plateau reached in 1972/75 and a turnover in 1988/89. The hot component contracted in radius at roughly constant luminosity from c. 1972 to 1986; during this phase it was the source of a strong stellar wind and therefore could not accrete any further material. By 1991 the luminosity of the nova remnant had decreased to a few per cent of the maximum (plateau) luminosity, and the hot wind had practically ceased. By 1995 the hot component start to accrete material from the Mira wind, as indicated by a general increase of the optical continuum and Balmer H I emission. The quiescent optical spectrum of RX Pup resembles the quiescent spectra of symbiotic recurrent novae, while the hot component luminosity is consistent with variable wind-accretion at a high rate, $\dot{M}_{\text {acc }} \sim 10^{-7} \mathrm{M}_{\odot} \mathrm{yr}^{-1}\left(\approx 1\right.$ per cent of $\left.\dot{M}_{\text {cool }}\right)$. RX Pup may be a recurrent nova; there is some evidence that a previous eruption occurred around 1894.

In the following we discuss results of optical and red spectroscopic observations of 


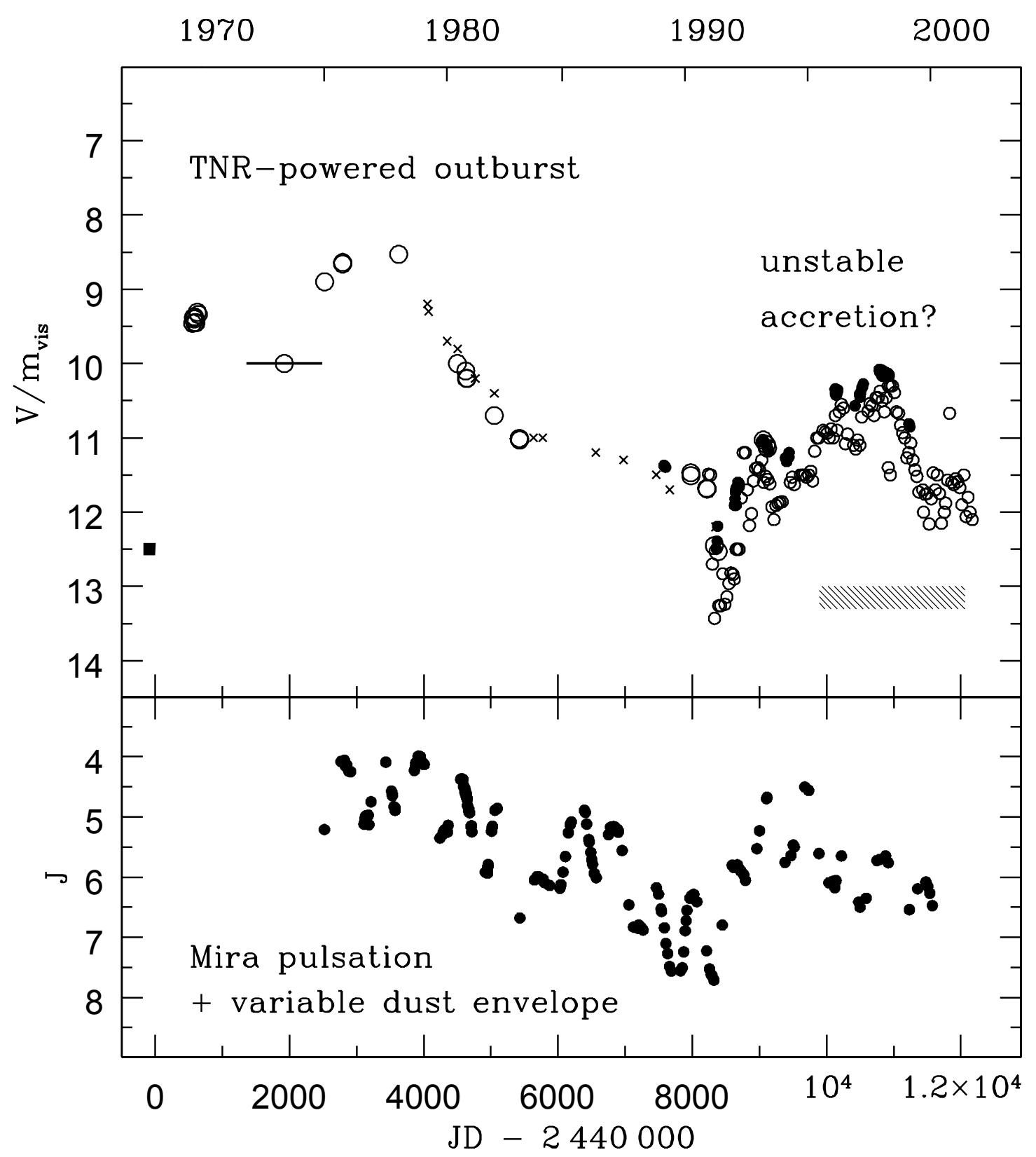

FIGURE 1. Optical and near- IR (J) light curves of RX Pup ([1]). In the $V / m_{\text {vis }}$ light curve, small open circles represent RASNZ observations; large open circles and dots published V data; crosses FES magnitudes. The optical light curve is dominated by the hot component activity whereas the $\mathrm{J}$ light curve is dominated by the Mira pulsations and variable obscuration of the Mira by circumstellar dust. The shaded area indicates the period of our spectroscopic observations discussed in Sec. 2.

RX Pup obtained during 1995-2001 with the REOSC echelle spectrograph at the 2.15m CASLEO telescope at San Juan, Argentina (see [1] for details), and low resolution CCD spectra obtained from the Vainu Bappu Observatory, India (see e.g. [2] for details). 

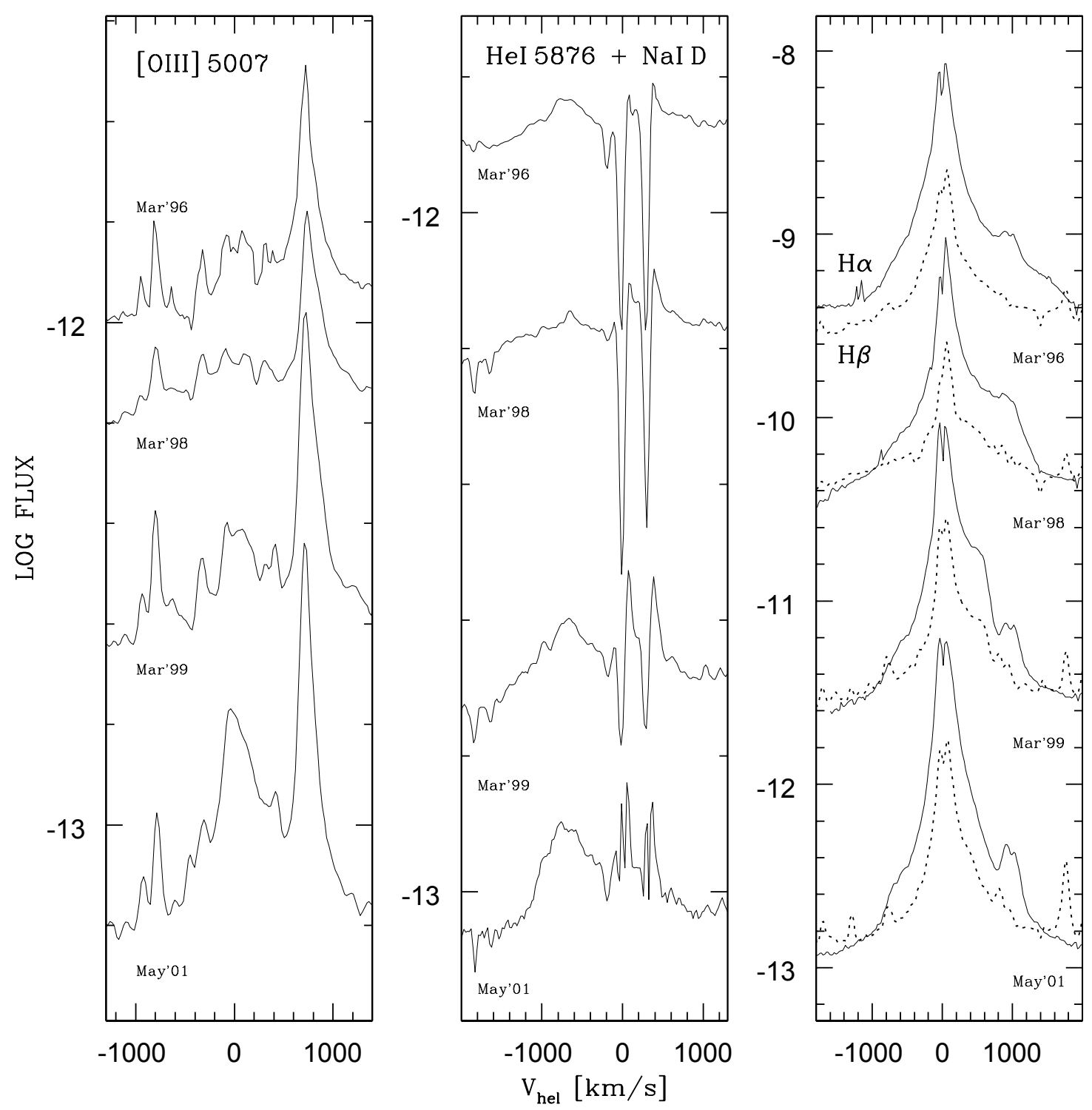

FIGURE 2. Evolution of emission line profiles in RX Pup in 1996-2001. The profiles are shifted vertically for better display.

\section{RESULTS AND DISCUSSION}

In the period covered by our spectroscopic observations, both components of RX Pup were active, although their changes were generally not correlated (Fig. 1). In particular, the near-IR flux was gradually decreasing in 1995-2000, suggesting that the Mira had entered a new obscuration phase ([3]). At the same time, the visual light curve revealed a small eruption with maximum in 1998. The lack of any molecular absorption bands in our spectra $(\lambda \leq 8600)$, as well as the absence of any Mira pulsations in the optical and red light curves (Fig.1; [1]]; [3]) implies that the hot component is responsible for all changes in the optical and red spectral range. 
The optical brightening was accompanied by significant spectroscopic changes. The changes were the most remarkable in Balmer H I lines: they were strong and narrow in 1988 - 91 ([1]) whereas in 1995 - 2001 they developed a prominent flattened red wing (Fig. 2). The broad asymmetric wings were also present in He I and near infrared Ca II triplet. The ionization level remained low, although the decline from the visual maximum in 1998 was associated with increase in [O III] and He I emission line fluxes. Our spectra also showed the presence of a variable blue continuum with $\mathrm{A} / \mathrm{F}$-type shell absorption lines. The colour temperature of the continuum is low, $\sim 6000-9000 \mathrm{~K}$ for $E_{\mathrm{B}-\mathrm{V}} \sim 0.8$, and it has a luminosity of $\sim 500-1000(d / 1.8 \mathrm{kpc})^{2} \mathrm{~L}_{\odot}$. On the other hand, the optical emission lines indicate a higher, $\sim 2.5-4 \times 10^{4} \mathrm{~K}$, temperature source with a roughly comparable luminosity. [3] also detected large changes in the degree of polarization in the optical and red spectral range, and found that the polarized component is radiation from the blue continuum source scattered in the dust envelope surrounding the Mira.

We find here a striking similarity between RX Pup and the quiescent symbiotic recurrent novae RS Oph and $\mathrm{T} \mathrm{CrB}$, as well as the hot component of $\mathrm{CH}$ Cyg during bright phases. In all these systems, the optical data indicate the presence of relatively cool F/A- (CH Cyg) or A/B-type source (RS Oph and T CrB), while the optical emission lines indicate a higher temperature source, with a roughly comparable luminosity ([円]; [5]; [2]). For example, in RS Oph the IR colours indicate the presence of an additional warm, $\geq 7000 \mathrm{~K}$, source whereas the IUE and optical spectra show an A-B type shell source with $L \sim 100-600 \mathrm{~L}_{\odot}$, accompanied by strong $\mathrm{H}$ I and moderate $\mathrm{He}$ I emission lines ([5], and references therein). Similar variable UV/optical continuum with $L \sim$ $40-100 \mathrm{~L}_{\odot}$ was observed in $\mathrm{T} \mathrm{CrB} \mathrm{([2]} \mathrm{and} \mathrm{references} \mathrm{therein).} \mathrm{Although} \mathrm{both} \mathrm{in} \mathrm{the}$ symbiotic recurrent novae, $\mathrm{T} \mathrm{CrB}$ and RS Oph, and in RX Pup, the average luminosity of the $\mathrm{B} / \mathrm{A} / \mathrm{F}$-type shell source is consistent with the accretion rate, $\dot{M} \geq 10^{-8} \mathrm{M}_{\odot} \mathrm{yr}^{-1}$, required by the theoretical models, the effective temperatures places the hot components far from the standard massive white dwarf tracks in the HR diagram. Simultaneously, the X-ray data suggest $1-2$ orders of magnitude lower accretion rates ([[1], and references therein). All three systems show similar quiescent behaviour: their hot components have highly variable luminosity and occasionally display blue-shifted absorption features, and broad asymmetric emission line wings (see also [1]; [2]; [5]).

We note here that similar A/F-type shell absorption spectrum was detected in AR Pav and other Z And-type symbiotic stars with multiple outburst activity(e.g. [6]). The blue absorption system in these systems traces the orbit of the hot component, and it is probably formed in a geometrically and optically thick accretion disc seen nearly edgeon and in a gas stream ([7]; [6], and references therein). Parenthetically, the timescales and amplitudes for their eruptions are very similar to the timescales and amplitudes of the hot component luminosity variations (high and low states) in RX Pup and other symbiotic recurrent novae between their nova outbursts, as well as in accretion-powered systems CH Cyg and MWC 560.

It is also interesting that the shell spectrum appears only during the late decline from the nova outburst. In particular, the optical/visual light curves from the outbursts of T CrB, RS Oph and RX Pup show more or less pronounced minima followed by a standstill (or secondary maximum) associated with the appearance of the shell spectrum. We suggest the minima are due to a decline in the hot component's luminosity after it passes 
the turnover in the HR diagram. The strong hot component wind during the plateau phase prevents accretion onto the hot component. Following the decline in luminosity the wind also ceases, and accretion of the material from the cool giant can be restored. We believe that the shell-type features and variable "false atmosphere" observed at quiescence together with the complex and broad emission lines originate from the accretion flow. The observed variability could be due to fluctuations in the mass-loss rate from the cool giant, the shell spectrum becomes stronger as $\dot{M}_{\text {cool }}$ increases and as a result $\dot{M}_{\text {acc }}$ increases. In the recurrent novae T CrB, RS Oph and the accretion- powered systems of $\mathrm{CH}$ Cyg and MWC 560, the brightening of the hot component (or high activity stage) is associated with the presence of flickering (e.g. [2]]). Such flickering, however, has not yet been detected in RX Pup.

Summarizing, the spectral development of RX Pup during the large outburst in 1969-1990 resembles that of symbiotic novae AG Peg, HM Sge and V1329 Cyg as well as of the symbiotic recurrent nova RS Oph ([1]), although these other systems seem to be evolving on very different timescales, possibly reflecting differences in the mass of the white dwarf component. At quiescence the hot component shows activity (high and low activity states) resembling that of symbiotic recurrent novae $\mathrm{T} \mathrm{CrB}$ and RS Oph, which is probably due to relatively high mass transfer/accretion rate, of order of $\sim 10^{-7} \mathrm{M}_{\odot} \mathrm{yr}^{-1}$. A high accretion rate, $\geq 10^{-8} \mathrm{M}_{\odot} \mathrm{yr}^{-1}$, is also required to account for the recurrence time, $\sim 80,80$ and $22 \mathrm{yr}$, for RX Pup, T CrB, and RS Oph, respectively.

\section{ACKNOWLEDGMENTS}

This study was partly supported by KBN research grant No. 5P03D 01920.

\section{REFERENCES}

1. Mikołajewska, J., Brandi, E., Hack, W., Whitelock, P.A., Barba, R., Garcia, L., Marang, F., 1999, MNRAS, 305, 190

2. Anupama, G.C., Mikołajewska, J., 1999, A\&A, 344, 177

3. Mikołajewska, J., Brandi, E., Garcia, L., Ferrer, O., W., Whitelock, P.A., Marang, F., 2001, in Szczerba R. et al., eds, Post-AGB Objects as a Phase of Stellar Evolution, Kluwer, 227

4. Mikołajewska, J., Selvelli, P.L., Hack, M., 1988, A\&A, 198, 150

5. Dobrzycka, D., Kenyon, S.J., Proga, D., Mikołajewska, J., Wade, R., 1996, AJ, 111, 2090

6. Quiroga, C., Mikołajewska, J., Brandi, , Ferrer, O., Garcia, L., 2002, A\&A, 387, 139

7. Mikołajewska, J., Kenyon, S.J., 1992, AJ, 103, 579 